\title{
Polyconvexity equals rank-one convexity for connected isotropic sets in $\mathrm{M} 2 \times 2$
}

\author{
Conti, S ; De Lellis, C ; Müller, S ; Romeo, M
}

\begin{abstract}
We give a short, self-contained argument showing that, for compact connected sets in M2x2 which are invariant under the left and right action of $\mathrm{SO}(2)$, polyconvexity is equivalent to rank-one convexity (and even to lamination convexity). As a corollary, the same holds for $\mathrm{O}(2)$-invariant compact sets. These results were first proved by Cardaliaguet and Tahraoui. We also give an example showing that the assumption of connectedness is necessary in the $\mathrm{SO}(2)$ case.
\end{abstract}

DOI: https://doi.org/10.1016/S1631-073X(03)00333-9

Posted at the Zurich Open Repository and Archive, University of Zurich

ZORA URL: https://doi.org/10.5167/uzh-21870

Journal Article

Originally published at:

Conti, S; De Lellis, C; Müller, S; Romeo, M (2003). Polyconvexity equals rank-one convexity for connected isotropic sets in $\mathrm{M} 2 \times 2$. Comptes Rendus Mathématique. Académie des Sciences. Paris, 337(4):233-238. DOI: https://doi.org/10.1016/S1631-073X(03)00333-9 


\section{Max-Planck-Institut für Mathematik in den Naturwissenschaften Leipzig}

Polyconvexity equals rank-one convexity for connected isotropic sets in $M^{2 \times 2}$

by

Sergio Conti, Camillo De Lellis, Stefan Müller, and Mario Romeo

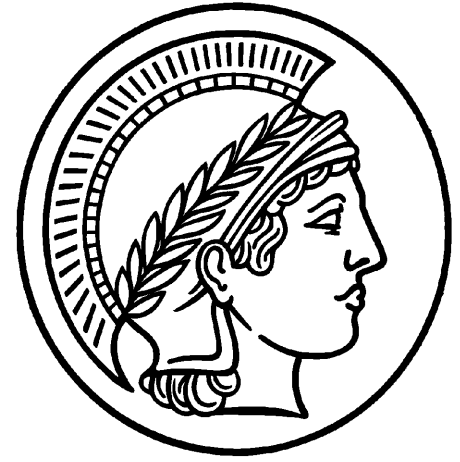





\title{
Polyconvexity equals rank-one convexity for connected isotropic sets in $\mathbb{M}^{2 \times 2}$
}

\author{
Sergio Conti, Camillo De Lellis, Stefan Müller and Mario Romeo \\ Max-Planck-Institute for Mathematics in the Sciences, \\ Inselstr. 22-26, 04103 Leipzig, Germany
}

NOVEMBER 6, 2002

\begin{abstract}
We give a short, self-contained argument showing that, for compact connected sets in $\mathbb{M}^{2 \times 2}$ which are invariant under the left and right action of $\mathrm{SO}(2)$, polyconvexity is equivalent to rank-one convexity (and even to lamination convexity). As a corollary, the same holds for $\mathrm{O}(2)$-invariant compact sets. These results were first proved by Cardaliaguet and Tahraoui. We also give an example showing that the assumption of connectedness is necessary in the $\mathrm{SO}(2)$ case.
\end{abstract}

Quasiconvexity was introduced by Morrey as a characterization of the energy densities $W$ which give rise to lower semicontinuous energy functionals $I[u]=\int W(\nabla u)$ defined on maps $u: \Omega \subset \mathbb{R}^{n} \rightarrow \mathbb{R}^{m}$ (in what follows we consider $n=m=2$ ). If $K$ is the compact set where $W$ attains its minimum, then its quasiconvex envelope $K^{q c}$ is the set of gradients of affine maps where the relaxation of $I$ attains its minimum [4]. From a physical point of view, $K^{q c}$ represents the set of macroscopic zero-energy deformation gradients which can be attained by an elastic solid.

A direct method to characterize $K^{q c}$ explicitely is missing. Inner and outer bounds, however, can often be derived. On the one hand, $K^{q c}$ is contained in the polyconvex hull $K^{p c}$, defined as the set of matrices which cannot be separated from $K$ by a polyconvex function $\left(\varphi: \mathbb{M}^{2 \times 2} \rightarrow \mathbb{R}\right.$ is polyconvex if $\varphi(X)=\psi(X$, $\operatorname{det} X)$ for some convex $\left.\psi: \mathbb{R}^{5} \rightarrow \mathbb{R}\right)$. By separation we mean that for every $X \notin K^{p c}$ there exists a polyconvex $\varphi$ such that $\varphi(X)>\varphi(Y)$ for any $Y \in K$. On the other hand, $K^{q c}$ contains the rank-one convex hull $K^{r c}$, defined as the set of matrices which cannot be separated by a rank-one convex function, i.e. a function which is convex along rank-one lines $t \rightarrow A+t a \otimes b$. The set $K^{r c}$ contains in particular the lamination-convex hull $K^{l c}$, defined as the intersection of all sets $H$ containing $K$ such that for every $X, Y \in H$ with $\operatorname{rank}(X-Y)=1$ the whole segment $[X, Y]$ belongs to $H$. Most known examples of quasiconvex sets are obtained by proving that in specific cases $K=K^{p c}=K^{l c}$. It is therefore interesting to investigate in which cases the latter property holds, i.e. to characterize classes of sets which are both lamination convex and polyconvex.

We focus here on isotropic materials in two dimensions. Isotropy is appropriate for example for elastomeric or polycristalline materials, and mathematically means that the energy density $W$ satisfies $W(X)=W\left(Q X Q^{\prime}\right)$ for all rotations $Q, Q^{\prime} \in \mathrm{SO}(2)$. Correspondingly, we say that the set $K \subset \mathbb{M}^{2 \times 2}$ is $\mathrm{SO}(2)$-invariant if $Q K Q^{\prime}=K$ for all $Q, Q^{\prime}$ in $\mathrm{SO}(2)$. We say that a set $K$ is polyconvex (rank-one convex, lamination convex) if $K=K^{p c}\left(K=K^{r c}\right.$, $K=K^{l c}$ resp.). We show that

Theorem 1. Let $K \subset \mathbb{M}^{2 \times 2}$ be compact, connected and $\mathrm{SO}(2)$-invariant. Then $K$ is lamination convex if and only if it is polyconvex. 
This result was first obtained by Cardaliaguet and Tahraoui [1] with the additional assumption that all matrices in $K$ have nonnegative determinant, and a rather long proof $[2,3]$. We give here a short, self-contained argument leading to the same result without the restriction on the determinant.

Isotropic functions can be naturally written in terms of the scalar parameters $\lambda_{1}(X)$ and $\lambda_{2}(X)$, which we define as the only real numbers $\left|\lambda_{1}\right| \leq \lambda_{2}$ such that $Q \operatorname{diag}\left(\lambda_{1}, \lambda_{2}\right) Q^{\prime}=X$ for some $Q, Q^{\prime} \in \mathrm{SO}(2)$. We remark that $\left|\lambda_{1}\right|$ and $\lambda_{2}$ are the singular values of $X$, i.e. the eigenvalues of $\left(X X^{T}\right)^{1 / 2}$. Hence $\lambda_{1}^{2}(X)+\lambda_{2}^{2}(X)=|X|^{2}:=\operatorname{Tr} X^{T} X$ and $\lambda_{1}(X) \lambda_{2}(X)=$ $\operatorname{det} X$. If $X \in \mathbb{M}^{2 \times 2}$, then $Y \in \mathrm{SO}(2) X \mathrm{SO}(2)$ if and only if $\lambda_{i}(X)=\lambda_{i}(Y)$. For an analysis of rank-one convexity for isotropic functions see [5] and references therein.

Lemma 2. Let $c \in \mathbb{R} \backslash\{0\}$. Then the functions

$$
\varphi_{c}^{ \pm}(X):=\lambda_{2}(X) \pm \lambda_{1}(X)-(\operatorname{det} X) / c
$$

are polyconvex. The same holds for $\varphi_{0}^{ \pm}(X):=-\operatorname{det}(X)$.

Proof. The lemma follows from the convexity of the functions $\lambda_{2} \pm \lambda_{1}$, which in turn is proved by the explicit computation

$$
\lambda_{2}(X) \pm \lambda_{1}(X)=\sqrt{|X|^{2} \pm 2 \operatorname{det} X}=\sqrt{\left(X_{11} \pm X_{22}\right)^{2}+\left(X_{21} \mp X_{12}\right)^{2}} .
$$

Remark 3. We observe that any $\mathrm{SO}(2)$-invariant polyconvex function can be written as supremum of linear combinations of the functions $\varphi_{c}^{ \pm}$, as can be seen by writing it first as supremum of polyaffine functions and then exploiting $\mathrm{SO}(2)$-invariance.

Before giving the detailed proof of Theorem 1, we illustrate how the argument can be visualized in the plane $\left(\lambda_{1}, \lambda_{2}\right)$. Suppose that $K$ is lamination convex. The level-sets of the functions $\varphi_{c}^{ \pm}$through a given matrix $A$ form a one-parameter family of hyperbolic arcs. These arcs are at the same time images of rank-one lines, hence if $A$ is not in $K$ they cannot intersect $K$ both 'before' and 'after' $A$. We divide each hyperbolic arc into two pieces, separated by the matrix $A$, and parametrize each ray with the vector $\mathbf{e} \in \mathbf{S}^{1}$ tangent to it in $A$ (see Figure 1). By continuity, the set $\gamma \subset \mathbf{S}^{1}$ of $\mathbf{e}$ for which the corresponding ray intersects the compact set $K$ is closed. Since $\gamma$ and $-\gamma$ are closed and disjoint subsets of the connected set $\mathbf{S}^{1}$, there is an $\mathbf{e} \in \mathbf{S}^{1}$ such that neither e nor -e lies in $\gamma$. The corresponding hyperbola $\{\bar{\varphi}=h\}$ does not intersect $K$, and since $K$ is assumed to be connected it lies on one side of it. Using Remark 4 we show below that either $K$ is a subset of $\{\bar{\varphi}<h\}$, or $K$ lies also on one side of $\{\operatorname{det}=\operatorname{det} A\}$. This will conclude the proof.

Remark 4. Suppose that $K$ is lamination convex. Then

$$
\text { if } X \in K \text {, then }\left\{Y: \operatorname{det}(Y)=\operatorname{det}(X), \lambda_{2}(Y) \leq \lambda_{2}(X)\right\} \subset K \text {. }
$$

To see this, consider the rank-one segment joining the matrices

$$
X_{ \pm}:=\left(\begin{array}{cc}
|\operatorname{det} X|^{1 / 2} & \pm \sqrt{|X|^{2}-2|\operatorname{det} X|} \\
0 & (\operatorname{det} X) /|\operatorname{det} X|^{1 / 2}
\end{array}\right) .
$$

Clearly $X_{ \pm} \in \mathrm{SO}(2) X \mathrm{SO}(2) \subset K$ and thus the whole rank-one segment $\left[X^{+}, X^{-}\right]$belongs to $K$. Along this segment the product of $\left|\lambda_{1}\right|$ and $\lambda_{2}$ is constant, and the sum of their squares is minimal when they are equal and maximal at the endpoints $X^{ \pm}$. Therefore for any $Y$ in the set (2) there exists $\tilde{Y} \in\left[X^{+}, X^{-}\right]$such that $\lambda_{i}(\tilde{Y})=\lambda_{i}(Y)$. 

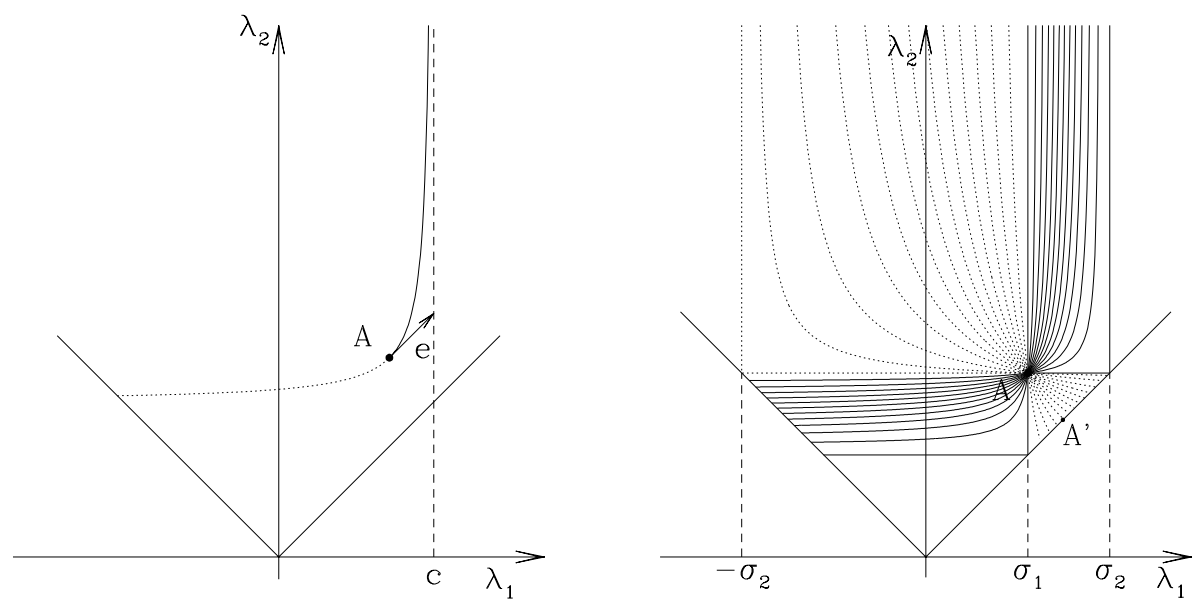

FiguRE 1. Left panel: one of the level sets $L_{c}$. The dotted part is $\Lambda\left(c+2 \sigma_{2}\right)$. Right panel: the family of closed sets $\Lambda(d)$. The dotted lines are the level sets of $\varphi_{c}^{-}$, the continuous are the level sets of $\varphi_{c}^{+}$. The point $(x, y)$ represents the set $\mathrm{SO}(2) \operatorname{diag}(x, y) \mathrm{SO}(2)$.

Proof of Theorem 1. Since det is affine on rank-one lines, a set of matrices which is polyconvex is also lamination convex, and one implication of the Theorem follows. To prove the other implication we need to show that for any $A \notin K$, there is a polyconvex $\varphi$ with $\varphi(A)>\max _{K} \varphi$. It is actually sufficient to show that, for any $K$ which satisfies the assumptions above,

if $A=\operatorname{diag}\left(\sigma_{1}, \sigma_{2}\right) \notin K$, with $0 \leq \sigma_{1} \leq \sigma_{2}$, there is $\varphi$ polyconvex s.t. $\varphi(A)>\max _{K} \varphi$.

Indeed suppose that (4) holds, and take $B \in \mathbb{M}^{2 \times 2} \backslash K$. Then there are $O_{1}, O_{2} \in \mathrm{O}(2)$ such that $A:=O_{1} B O_{2}$ has the form above. The set $K^{\prime}:=O_{1} K O_{2}$ is still compact, connected, lamination convex and $\mathrm{SO}(2)$-invariant. By (4) there is a polyconvex function $\varphi^{\prime}$ which separates $A$ from $K^{\prime}$. Then $\varphi(X):=\varphi^{\prime}\left(O_{1} X O_{2}\right)$ is polyconvex and separates $B$ from $K$.

We now start proving (4). If $\sigma_{1}=\sigma_{2}$, we claim that $\{Y: \operatorname{det} Y=\operatorname{det} A\}$ does not intersect $K$. Otherwise there exists $X \in K$ with $\operatorname{det} X=\operatorname{det} A$ and thus $\lambda_{2}(X) \geq \sigma_{2}=\sigma_{1} \geq \lambda_{1}(X)$. Hence (2) yields that $A \in K$, a contradiction. Thus, the connectedness of $K$ implies that either $\max _{K} \operatorname{det}<\operatorname{det} A$ or $\max _{K}-\operatorname{det}<-\operatorname{det} A$. Since $\pm \operatorname{det}$ are both polyconvex we are done.

If instead $\sigma_{2}>\sigma_{1}$, we show below that at least one of the level sets

$$
L_{c}:= \begin{cases}\left\{X \mid \varphi_{c}^{-}(X)=\varphi_{c}^{-}(A)\right\} & \text { for } c \in\left[-\sigma_{2}, \sigma_{1}[\right. \\ \left\{X \mid \varphi_{c}^{+}(X)=\varphi_{c}^{+}(A)\right\} & \text { for } c \in\left[\sigma_{1}, \sigma_{2}\right]\end{cases}
$$

does not intersect $K$ (in the $\left(\lambda_{1}, \lambda_{2}\right)$ plane with $\left|\lambda_{1}\right| \leq \lambda_{2}, L_{c}$ is an arc of hyperbola with vertical asymptote $\lambda_{1}=c$, see Figure 1). This implies immediately the result. Indeed, let $\bar{\varphi}$ be the one of the $\varphi_{c}^{ \pm}$which generates this level set. Since $K$ is compact and connected then either $\max _{K} \bar{\varphi}<\bar{\varphi}(A)$ or $\min _{K} \bar{\varphi}>\bar{\varphi}(A)$. In the first case our proof is finished. In the 
second case, consider $A^{\prime}=\left(\sigma_{1} \sigma_{2}\right)^{1 / 2} \mathrm{Id}$. Since $\varphi_{c}^{ \pm}(A) \geq \varphi_{c}^{ \pm}\left(A^{\prime}\right)$ for all $c$, the matrix $A^{\prime}$ is not in $K$. Then by the argument above either det or - det separates $A^{\prime}$, and hence $A$, from $K$.

We now come to the core of the proof, which consists in showing that one of the level sets (5) does not intersect $K$. For $c \neq \pm \sigma_{2}$ we split any of the $L_{c}$ into two pieces (see Figure 1),

$$
L_{c}^{>}:=L_{c} \cap\left\{\lambda_{2}(X) \geq \sigma_{2}\right\} \quad \text { and } \quad L_{c}^{<}:=L_{c} \cap\left\{\lambda_{2}(X) \leq \sigma_{2}\right\} .
$$

One basic remark is that for any $c \in]-\sigma_{2}, \sigma_{2}[$,

$$
\text { either } L_{c}^{>} \cap K=\emptyset \text { or } L_{c}^{<} \cap K=\emptyset \text {. }
$$

Indeed, if $B \in L_{c}^{>}$and $C \in L_{c}^{<}$, we can find a rank-one segment between an element of $\mathrm{SO}(2) B \mathrm{SO}(2)$ and an element $\mathrm{SO}(2) C \mathrm{SO}(2)$ which contains $A$. To show this we distinguish two cases. If $\sigma_{1} \leq c<\sigma_{2}$, we choose $s \in[0,1]$ such that $s \sigma_{1}+(1-s) \sigma_{2}=c$, and define

$$
A(t):=A+t\left(\begin{array}{cc}
1-s & \sqrt{s(1-s)} \\
\sqrt{s(1-s)} & s
\end{array}\right) \quad \begin{aligned}
t_{B} & :=\lambda_{2}(B)+\lambda_{1}(B)-\sigma_{2}-\sigma_{1} \\
t_{C} & :=\lambda_{2}(C)+\lambda_{1}(C)-\sigma_{2}-\sigma_{1}
\end{aligned}
$$

It is easy to check that $\left(\lambda_{2}+\lambda_{1}\right)^{2}(A(t))=|A(t)|^{2}+2 \operatorname{det} A(t)=\left(\sigma_{2}+\sigma_{1}+t\right)^{2}, t_{B} \geq 0, t_{C} \leq 0$, and $\varphi_{c}^{+}(A(t))=\varphi_{c}^{+}(A)=\varphi_{c}^{+}\left(\operatorname{diag}\left(\sigma_{1}, \sigma_{2}\right)\right)$. Hence $\lambda_{i}\left(A\left(t_{B}\right)\right)=\lambda_{i}(B), \lambda_{i}\left(A\left(t_{C}\right)\right)=\lambda_{i}(C)$. If instead $-\sigma_{2}<c<\sigma_{1}$ we reason in the same way choosing $s$ such that $-s \sigma_{2}+(1-s) \sigma_{1}=c$ and

$$
A(t):=A+t\left(\begin{array}{cc}
-s & -\sqrt{s(1-s)} \\
\sqrt{s(1-s)} & 1-s
\end{array}\right) \quad \begin{aligned}
t_{B} & :=\lambda_{2}(B)-\lambda_{1}(B)-\sigma_{2}+\sigma_{1} \\
t_{C} & :=\lambda_{2}(C)-\lambda_{1}(C)-\sigma_{2}+\sigma_{1} .
\end{aligned}
$$

and considering that $\left(\lambda_{2}-\lambda_{1}\right)^{2}(A(t))=\left(\sigma_{2}-\sigma_{1}+t\right)^{2}$.

The second basic remark is that for $c \in]-\sigma_{2}, \sigma_{2}[$ and $\square \in\{>,<\}$, the following holds:

$$
\text { if } X_{n} \in L_{c_{n}}^{\square}, c_{n} \rightarrow c \text {, and } X_{n} \rightarrow X \text {, then } X \in L_{c}^{\square} \text {. }
$$

We now extend and reparametrize $L_{c}^{>}$and $L_{c}^{<}$to obtain an $\mathbf{S}^{1}$-parameter family of sets $\Lambda(d)$, for $d \in\left[-\sigma_{2}, 3 \sigma_{2}\right]$, such that the properties (7) and (8) still hold. For $|c|<\sigma_{2}$ we set $\Lambda(c):=L_{c}^{>}$and $\Lambda\left(2 \sigma_{2}+c\right):=L_{c}^{<}$. The remaining sets are

$$
\Lambda\left(\sigma_{2}\right):=L_{\sigma_{2}} \cap\left\{\lambda_{1}(X) \geq \sigma_{1}\right\} \text { and } \Lambda\left(-\sigma_{2}\right)=\Lambda\left(3 \sigma_{2}\right):=L_{-\sigma_{2}} \cap\left\{\lambda_{1}(X) \leq \sigma_{1}\right\} .
$$

To better visualize the $\mathbf{S}^{1}$ family one could reparametrize it replacing $d$ with the (oriented) tangent vector to the sets $\Lambda$ in the point $\left(\sigma_{1}, \sigma_{2}\right)$ in the $\left(\lambda_{1}, \lambda_{2}\right)$ plane (see Figure 1$)$.

We call $\tilde{K}$ the set of those $d \in\left[-\sigma_{2}, 3 \sigma_{2}\right]$ such that $\Lambda(d)$ intersects $K$. In view of $(8)$, and its obvious extension to the limits cases $c= \pm \sigma_{2}, \tilde{K}$ is closed, and in view of (7), $\tilde{K}$ and $\left(\tilde{K}+2 \sigma_{2}\right)$ are disjoint. Hence their union cannot cover all of the connected set $\left[-\sigma_{2}, 3 \sigma_{2}\right]$, and there is $d \in\left[-\sigma_{2}, \sigma_{2}\right]$ such that both $\Lambda(d)$ and $\Lambda\left(d+2 \sigma_{2}\right)$ do not intersect $K$. Since $L_{d}$ is contained in $\Lambda(d) \cup \Lambda\left(d+2 \sigma_{2}\right)$ the proof is finished.

Corollary 5. Let $K \subset \mathbb{M}^{2 \times 2}$ be compact and $\mathrm{O}(2)$-invariant. Then $K$ is lamination convex if and only if it is polyconvex.

Proof. The claim follows from the fact that any $\mathrm{O}(2)$-invariant nonempty lamination convex set is connected and $\mathrm{SO}(2)$-invariant. To show that $K$ is connected, we remark that if $X=\operatorname{diag}\left(\mu_{1}, \mu_{2}\right) \in K$, then the rank-one segment connecting $X$ with $X^{\prime}=\operatorname{diag}\left(-\mu_{1}, \mu_{2}\right)$ is in $K$, and hence also the one connecting $\operatorname{diag}\left(0, \mu_{2}\right)$ with $\operatorname{diag}\left(0,-\mu_{2}\right)$, which contains 0 . 


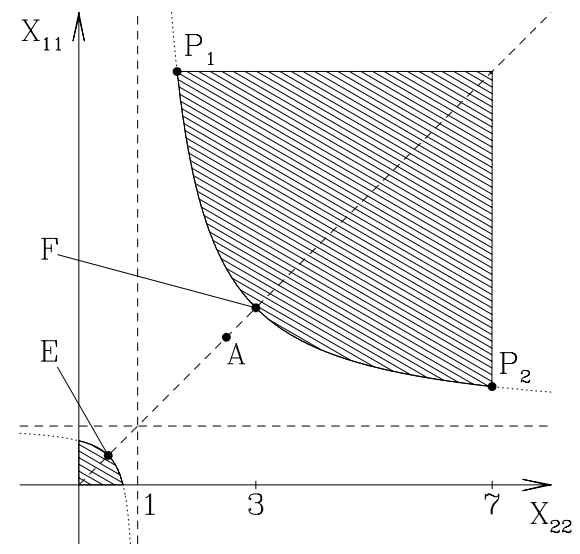

Figure 2. Intersection of the set $K$ defined in (9) with the diagonal matrices with nonnegative entries. The matrices $A, E, F, P_{1}$ and $P_{2}$ entering (10) are also shown.

We now show with an example that if $K$ is not connected, with the remaining assumptions of Theorem 1 still holding, then rank-one convexity (and hence lamination convexity) does not imply polyconvexity. Consider the function

$$
f(X):= \begin{cases}4\left(\lambda_{2}+\lambda_{1}-1-\lambda_{1} \lambda_{2}\right)(X) & \lambda_{1}(X) \leq 1 \\ \frac{1}{4}\left(\lambda_{2}+\lambda_{1}-1-\lambda_{1} \lambda_{2}\right)(X) & \lambda_{1}(X)>1,\end{cases}
$$

which is rank-one convex by Lemma 3.1 of [6]. Define

$$
K:=\left\{X: f(X) \leq-1, \lambda_{2}(X) \leq 7\right\}
$$

and $A=\operatorname{diag}(5 / 2,5 / 2)$. The set $K$ is rank-one convex, compact, $\mathrm{SO}(2)$-invariant, and does not contain $A$ (see Figure 2). We now show that there is no polyaffine function $\psi$, and hence no polyconvex function, which separates $A$ from $K$. This implies that $K$ is not polyconvex. Consider the four matrices $E=\operatorname{diag}(1 / 2,1 / 2), F=\operatorname{diag}(3,3), P_{1}=\operatorname{diag}(7,5 / 3)$, and $P_{2}=\operatorname{diag}(5 / 3,7)$ (all of which belong to $K$ ). It is a simple check that the inequalities

$$
\psi(A)>\psi(E), \quad \psi(A)>\psi(F), \quad 2 \psi(A)>\psi\left(P_{1}\right)+\psi\left(P_{2}\right)
$$

are incompatible. To see this, write $\psi(X)=B: X-c \operatorname{det} X+d$, with $B \in \mathbb{M}^{2 \times 2}$ and $c, d \in \mathbb{R}$ (and $B: X=\operatorname{Tr} B^{T} X$ ). Setting $b:=\operatorname{Tr} B / 2$, the inequalities above become

$$
5 b-\frac{25}{4} c>b-\frac{1}{4} c, \quad 5 b-\frac{25}{4} c>6 b-9 c, \quad 5 b-\frac{25}{4} c>\frac{26}{3} b-\frac{35}{3} c .
$$

It is easy to see that they are incompatible. Graphically, this corresponds to the fact that, if $A$ is sufficiently close to $F$, any hyperbola which separates $A$ from $P_{1}, F$ and $P_{2}$ is very close to the one which contains the latter three points (see Figure 2). Then, its second branch does not separate $A$ from $E$. 
Acknowledgements. This work was partially supported by the EU Research Training Network Homogenization and Multiple Scales, contract HPRN-CT-2000-00109, the EU TMR network Phase Transitions in Crystalline Solids, contract FMRX-CT98-0229, and by the DFG Schwerpunktprogramm 1095 Analysis, Modeling and Simulation of Multiscale Problems.

\section{REFERENCES}

[1] P. Cardaliaguet and R. Tahraoui, Sur l'équivalence de la 1-rang convexité et de la polyconvexité des ensembles isotropiques de $\mathbb{R}^{2 \times 2}$, C. R. Acad. Sci. Paris, Sér. I 331 (2000), 851-856.

$[2] \longrightarrow$, Equivalence between rank-one convexity and polyconvexity for isotropic sets of $\mathbb{R}^{2 \times 2}$ (part I), Nonlin. Anal. 50 (2002), 1179-1199.

[3] _ Equivalence between rank-one convexity and polyconvexity for isotropic sets of $\mathbb{R}^{2 \times 2}$ (part II), Nonlin. Anal. 50 (2002), 1201-1239.

[4] S. Müller, Variational models for microstructure and phase transitions, Calculus of variations and geometric evolution problems (F. Bethuel et al., eds.), Springer Lecture Notes in Math. 1713, Springer, Berlin, 1999, pp. 85-210.

[5] M. Šilhavý, Rotationally invariant rank 1 convex functions, Appl. Math. Optim. 44 (2001), 1-15.

[6] V. Šverák, Examples of rank-one convex functions, Proc. Roy. Soc. Edin. Sect. A 114 (1990), 237-242. 